\title{
Blood Pressure Variability and Atrial Fibrillation in Patients with Acute ST Segment Elevation Myocardial Infarction: The Relation with Left Atrial Electromechanical Delay - A 1-Year Follow-Up Study
}

\author{
Ragab A. Mahfouz Mohamed El-Shetry Abdelfattah Frere \\ Mohamed Safwat \\ Cardiology Department, Zagazig University Hospital, Zagazig, Egypt
}

\section{Keywords}

Blood pressure variability · Atrial fibrillation · ST segment myocardial infarction · Left atrial electromechanical delay

\begin{abstract}
Purpose: To investigate the association between 24 -h blood pressure variability (BPV) and atrial electromechanical delay (EMD) in patients with ST segment elevation myocardial infarction (STEMI) who developed new-onset atrial fibrillation (NOAF). Materials and Methods: A total of 175 STEMI patients (age $56.6 \pm 10.5$ years) who underwent primary percutaneous coronary intervention were subjected to in-hospital 24-h ambulatory BP monitoring, comprehensive echocardiography, and assessment of atrial EMD. The parameters of BPV analyzed were: (a) 24-h standard deviation (SD), (b) the coefficient of variation, and (c) the average of the daytime and nighttime SDs weighted for the duration of the daytime and nighttime interval $\left(S D_{d n}\right)$. Results: Based on the median of $B P V$ index $\left(S D_{d n}\right)=9.5 \mathrm{~mm} \mathrm{Hg}$ of all participants, patients were stratified into low and high variability groups $\left(\mathrm{SD}_{\mathrm{dn}}: 7.1 \pm 1.5\right.$ vs.13.5 \pm $2.9 ; p<0.001)$. Of the 175 patients with STEMI, 29 (16.7\%) patients developed NOAF; $26(28.9 \%)$ were in the high variability group and $3.5 \%$ were in the low variability group $(p<0.001)$. Echocardiographic data showed that the left atrial volume index $(p<0.01)$ and $\mathrm{E} / \mathrm{e}^{\prime}$ ratio $(p<0.001)$ were significantly higher in patients with high BPV. Inter and intra-atrial EMD were significantly increased in the high variability group compared to the low variability group $(p<0.001)$. With multiple linear analysis, there was significant correlation between $\mathrm{SD}_{\mathrm{dn}}$ and intra-left
\end{abstract}


Mahfouz et al.: Blood Pressure Variability and Atrial Fibrillation in STEMI

atrial and inter-atrial EMD ( $p<0.001$ and $<0.01$, respectively). Cox regression analysis revealed that $S D_{\mathrm{dn}}$ and intra-atrial EMD were independent predictors for NOAF in patients with STEMI $(\mathrm{OR}=3.75$ and 02.72 , respectively; $p<0.001)$. ROC analysis revealed that $\mathrm{SD}_{\mathrm{dn}} \geq 12.8$ was the optimal cut-off value for predicting NOAF during follow-up. Conclusions: Short-term BPV was associated with NOAF during the 1-year follow-up in patients with STEMI. In addition, BPV was correlated significantly with atrial EMD. Herein, BPV was predicted to be an early predictor of NOAF in patients with STEMI.

(c) 2020 S. Karger AG, Base

\section{Introduction}

New-onset atrial fibrillation (NOAF) is a frequent arrhythmia in the setting of ST segment elevation myocardial infarction (STEMI, and has a negative impact on clinical outcome [1]. The pathogenesis of the occurrence of atrial fibrillation following myocardial infarction is multifaceted. It may include the risk factors for ischemic heart disease themselves, associated comorbidities, hemodynamic instability, severity of the coronary lesion, and inadequate perfusion after thrombolytics or after primary percutaneous coronary intervention [2,3].

Blood pressure variability (BPV) is usually stratified into short-term (minutes to hours) and long-term BPV (days to months), while beat-to-beat oscillation is termed very-shortterm BPV [4]. It is thought to be associated with cardiac events in hypertensive patients and in STEMI cases [5-7].

Atrial electromechanical delay (EMD) is an important marker of an arrhythmogenic substrate in subjects with atrial fibrillation [8, 9]. EMD is the time interval from the start of the P wave on the surface ECG to the beginning of the A wave of the mitral annulus on tissue Doppler imaging (the PA interval). In our research, we investigated the relationship between 24-h BPV and atrial EMD, and their association with NOAF in STEMI patients.

\section{Subjects and Methods}

A total of 189 STEMI patients who underwent successful primary percutaneous coronary intervention were recruited for the study. STEMI was defined as $\geq 2 \mathrm{~mm}(0.2 \mathrm{mV})$ in males or $\geq 1.5 \mathrm{~mm}$ new ST elevation in V2-V3 leads, or $\geq 1 \mathrm{~mm}(0.1 \mathrm{mV})$ in any other contiguous leads [10]. In addition, we considered new or supposed new LBBB to be equal to STEMI [11]. We Successful myocardial infarction was considered as Thrombolysis in Myocardial Infarction (TIMI) grade 3 flow with less than 30\% residual stenosis. Patients with known paroxysmal atrial fibrillation at any time before index event, cardiogenic shock (as they received inotropes which may trigger atrial fibrillation), history of previous revascularization, or poor echocardiographic window were excluded. Patients with inflammatory conditions, chronic kidney diseases, or stroke were also excluded. Fourteen patients who did not complete follow-up were excluded, leaving a final number of 175 patients included in the study analysis.

\section{Ambulatory BP Monitoring}

All patients underwent ambulatory BP monitoring (ABPM) for $24 \mathrm{~h}$ within 7 days of discharge, using Mobil-O Graph (IEM GmbH, Stolberg, Germany). Measurements were scheduled to be recorded at 30-min intervals during the day (6 a.m.-11 p.m.) and at 45-min intervals during the night (11 p.m.-6 a.m.) [12]. The mean 24-h systolic (SBP) and diastolic (DBP) BP were obtained. Short-term BP variability was used as the standard deviation (SD), coefficient of variation (CV), and the average of the daytime and nighttime SDs weighted for the duration of the daytime and nighttime interval $\left(\mathrm{SD}_{\mathrm{dn}}\right)$. The $\mathrm{SD}_{\mathrm{dn}}$ is the mean of the wake and sleep SD values corrected for the number of hours included in each of these two periods, according to the following formula: $\mathrm{SD}_{\mathrm{dn}}=([$ day $\mathrm{SD} \times$ hours included in the daytime $]+$ [night $\mathrm{SD} \times$ hours included in the nighttime])/(hours included in daytime + nighttime) [13]. 


\begin{tabular}{l|l}
\hline Pulse 2020;8:57-65 \\
\hline DOI: 10.1159/000507792 & $\begin{array}{l}\text { (c) 2020 S. Karger AG, Basel } \\
\text { www.karger.com/pls }\end{array}$ \\
\hline
\end{tabular}

Mahfouz et al.: Blood Pressure Variability and Atrial Fibrillation in STEMI

Table 1. Baseline characteristics of the study population

\begin{tabular}{|c|c|c|c|c|}
\hline & $\begin{array}{l}\text { All patients } \\
(n=175)\end{array}$ & $\begin{array}{l}\text { Low BPV } \\
(n=85)\end{array}$ & $\begin{array}{l}\text { High BPV } \\
(n=90)\end{array}$ & $p$ value \\
\hline Age, years & $56.6 \pm 10.5$ & $54.5 \pm 11.3$ & $57.8 \pm 10.5$ & 0.34 \\
\hline Male & $118(67.4)$ & $55(64.7)$ & $63(70)$ & 0.29 \\
\hline Body mass index, $\mathrm{kg} / \mathrm{m}^{2}$ & $24.8 \pm 0.9$ & $24.3 \pm 0.9$ & $25.1 \pm 1.1$ & 0.57 \\
\hline Smoking & 73 (41.7) & $35(41.2)$ & $38(42)$ & 0.34 \\
\hline Diabetes mellitus & $80(45.7)$ & $37(43.5)$ & $43(47.8)$ & 0.46 \\
\hline Hypertension & $86(49.1)$ & $39(45.9)$ & $47(55.6)$ & 0.06 \\
\hline Dyslipidemia & $54(31)$ & $29(34)$ & $25(28)$ & 0.41 \\
\hline Family history & $40(20)$ & $15(17.6)$ & $20(22)$ & 0.30 \\
\hline Systolic BP, mm Hg & $130 \pm 31$ & $130 \pm 25$ & $132 \pm 35$ & 0.16 \\
\hline Diastolic BP, mm Hg & $79 \pm 21$ & $80 \pm 19$ & $79 \pm 25$ & 0.55 \\
\hline Pulse rate, beats/min & $86 \pm 23$ & $88 \pm 15$ & $89 \pm 22$ & 0.41 \\
\hline \multicolumn{5}{|l|}{ Site of infarction } \\
\hline Anterior wall myocardial infarction & 103 & 51 & 52 & 0.48 \\
\hline Inferior wall myocardial infarction & 59 & 28 & 31 & 031 \\
\hline Anterior + inferior & 13 & 7 & 6 & 0.35 \\
\hline \multicolumn{5}{|l|}{ Medications } \\
\hline$\beta$-Blocker & $99(56.6)$ & $46(54)$ & $53(58.9)$ & 0.57 \\
\hline Calcium channel blockers & $41(23.4)$ & $19(22.4)$ & $22(24.4)$ & 0.54 \\
\hline ACEIs & $35(20)$ & $16(18.8)$ & $19(21)$ & 0.78 \\
\hline ARBs & $48(27)$ & $22(25.9)$ & $26(28.9)$ & 0.60 \\
\hline Diuretics & $39(22)$ & $18(21)$ & $21(23)$ & 0.65 \\
\hline Nitroglycerines & $34(19.4)$ & $15(17.6)$ & $19(21)$ & 0.51 \\
\hline
\end{tabular}

Data are presented as mean \pm SD or $n(\%)$, as appropriate.

Calculation of BPV Indices

$B P V$ Index. BPV index was defined as the weighted SD of 24-h BP, the awake and sleep period BP for both SBP and DBP.

Dipping Status. Patients with an average night BP drop of 10-20\% are considered normal dippers, whilst nondippers are defined as those with an average drop of BP $<10 \%$. Subjects with an average drop of BP $>$ $20 \%$ are considered extreme dippers. Extreme dippers are patients with an average night BP decrease of $>$ $20 \%$ of the average daytime BP, whereas reversed dippers are those with an average sleep period BP higher than the average awake period BP [14].

BP Morning Surge. Subjectively, the morning period is the duration from 6:00 a.m. to 10:00 a.m. BP morning surge was calculated as the average of $\mathrm{BP}$ obtained at the first $2 \mathrm{~h}$ after waking minus the average $\mathrm{BP}$ readings during the night (as the average BP of 3 readings [15]).

\section{Transthoracic Echocardiography}

All participates underwent transthoracic echocardiographic evaluation using a commercially available cardiac ultrasound scanner (System Five; GE Vingmed Ultrasound, Horten, Norway) and 2.5- to 3.5-MHz transducers. Left ventricular (LV) dimensions, LV mass index, relative wall thickness, and left atrial dimensions as well as all conventional and tissue Doppler parameters were obtained and recorded. LV ejection fraction (EF\%), left atrial volume index (LAVi) was determined by dividing left atrial volume by estimated body surface area. E/A ratio, mean mitral annular wave velocity (é), and mean E/e' were calculated [16].

\section{Estimation of Atrial EMD}

The time interval from the onset of the P wave on the surface ECG to the beginning of the A wave was considered the atrial EMD (PA interval). A wave was recorded at the lateral mitral annulus, the septal mitral annulus as well as the tricuspid annulus. Three cycles were recorded and the mean was obtained [17].

- $\quad$ Intra-right atrial conduction time = septal mitral annular PA minus tricuspid annular PA.

- Intra-left atrial conduction time = lateral mitral annular PA minus septal mitral annular PA.

- $\quad$ Inter-atrial conduction time = lateral mitral annular PA minus tricuspid annular PA. 


\begin{tabular}{l|l}
\hline Pulse 2020;8:57-65 \\
\hline DOI: 10.1159/000507792 & $\begin{array}{l}\text { @ 2020 S. Karger AG, Basel } \\
\text { www.karger.com/pls }\end{array}$ \\
\hline
\end{tabular}

Mahfouz et al.: Blood Pressure Variability and Atrial Fibrillation in STEMI

Table 2. Blood pressure variability indices in the study groups

\begin{tabular}{|c|c|c|c|c|}
\hline ABPM parameters & $\begin{array}{l}\text { All patients } \\
(n=175)\end{array}$ & $\begin{array}{l}\text { Low BPV } \\
(n=85)\end{array}$ & $\begin{array}{l}\text { High BPV } \\
(n=90)\end{array}$ & $p$ value \\
\hline SD of day systolic readings & $11.5 \pm 4.3$ & $8.3 \pm 2.5$ & $13.9 \pm 5.1$ & $<0.001^{*}$ \\
\hline SD of day diastolic readings & $7.5 \pm 2.8$ & $7.1 \pm 1.5$ & $8.6 \pm 3.3$ & 0.13 \\
\hline SD of night systolic readings & $10.3 \pm 4.7$ & $8.3 \pm 2.9$ & $12.9 \pm 4.1$ & $0.002^{*}$ \\
\hline SD of night diastolic readings & $7.5 \pm 2.7$ & $6.1 \pm 1.5$ & $9.2 \pm 3.2$ & $<0.01^{*}$ \\
\hline SD of total systolic readings & $11.9 \pm 3.9$ & $9.3 \pm 2.9$ & $14.0 \pm 3.4$ & $<0.001^{*}$ \\
\hline SD of total diastolic readings & $8.2 \pm 2.7$ & $6.5 \pm 1.4$ & $9.1 \pm 2.5$ & $<0.01^{*}$ \\
\hline CV SBP, $\%$ & $9.8 \pm 6.1$ & $8.9 \pm 3.3$ & $11.5 \pm 7.6$ & $<0.003^{*}$ \\
\hline CV DBP, \% & $4.1 \pm 2.0$ & $3.9 \pm 2.0$ & $4.2 \pm 1.9$ & 0.23 \\
\hline Nondippers & $72(41)$ & $13(15)$ & $59(65.5)$ & $<0.01$ \\
\hline SBP Morning surge, mm Hg & $39 \pm 8$ & $13 \pm 4$ & $48 \pm 11$ & $<0.001^{*}$ \\
\hline DBP Morning surge, $\mathrm{mm} \mathrm{Hg}$ & $13 \pm 4$ & $10 \pm 3$ & $28 \pm 7$ & $<0.01^{*}$ \\
\hline \multicolumn{5}{|l|}{ BPV index } \\
\hline Weighted systolic SD day-night & $10.2 \pm 3.4$ & $7.1 \pm 1.5$ & $13.7 \pm 2.9$ & $<0.001^{*}$ \\
\hline Weighted diastolic SD day-night & $3.1 \pm 1.5$ & $2.9 \pm 0.9$ & $3.3 \pm 1.6$ & 0.09 \\
\hline
\end{tabular}

Data are presented as mean \pm SD or $n(\%)$, as appropriate. APBM, ambulatory BP monitoring; BP, blood pressure; SD, standard deviation; CV, coefficient variability; BPV, blood pressure variability. * Significance between high and low BPV.

Statistical Analysis

Categorical variables are presented as medians or means \pm SDs, as appropriate. Comparisons between groups were obtained using the $\chi^{2}$ test or Fisher's exact test, while continuous and normally distributed variables were compared by two-tailed unpaired $t$ tests. Correlation tests were performed using the Spearman correlation coefficient test. Based on the median of $\mathrm{BPV}$ index $\left(\mathrm{SD}_{\mathrm{dn}}\right)=9.5 \mathrm{~mm} \mathrm{Hg}$, participants were stratified into two groups: low variability group and high variability group. Patients were followed up for $14 \pm 3$ months and the end point was the occurrence of atrial fibrillation. Univariable and multivariable regression analyses were carried out to characterize predictors of NOAF during follow-up. A receiver operating characteristic (ROC) curve was used to characterize the cut-off value of BPV index that predicts the occurrence of NOAF. SPSS version 16.0 (SPSS Inc) was used to perform all statistical analyses.

\section{Results}

Of the 175 patients who completed follow-up, 29 (16.7\%) patients developed NOAF. Importantly, patients with high BPV had higher prevalence compared to those with low BPV (26 [28.9\%] vs. 3 [3.5\%], $p<0.001$ ). Table 1 presents the demographic characteristics and ABPM parameters of patients with low versus high BPV. All demographic data were comparable among both groups. Only hypertension was more common in the high variability group, but the difference was not significant.

As seen in Table 2, the results show that all ABPM parameters were significantly increased in the high variability group compared with the low variability group, except for CV DBP and weighted diastolic $\mathrm{SD}_{\mathrm{dn}}$. CV SBP was $11.5 \pm 7.6$ in the high variability group versus $8.9 \pm 3.3$ in the low variability group $(p<0.003)$, and weighted systolic $\mathrm{SD}_{\mathrm{dn}}$ was $13.7 \pm 2.9$ versus 7.1 \pm 1.5 , respectively $(p<0.001)$. In addition, SBP and DBP morning surges were increased $(p<$ 0.001 and $p<0.01$, respectively). Furthermore, the percentage of nondippers was higher in those with high BP variability $(p<0.01)$.

Table 3 illustrates the echocardiographic characteristics of patients with low versus those with high BPV index. As seen, LAVi $(p<0.01)$ and E/e" $(p<0.01)$ were significantly 


\begin{tabular}{l|l}
\hline Pulse 2020;8:57-65 \\
\hline DOI: 10.1159/000507792 & $\begin{array}{l}\text { @ 2020 S. Karger AG, Basel } \\
\text { www.karger.com/pls }\end{array}$ \\
\hline
\end{tabular}

Table 3. Echocardiographic data of patients with low versus high blood pressure variability

\begin{tabular}{lccc}
\hline & $\begin{array}{l}\text { Low variability } \\
(n=85)\end{array}$ & $\begin{array}{l}\text { High variability } \\
(n=90)\end{array}$ & $p$ value \\
\hline LVMI, g/m² & $91.5 \pm 13.6$ & $93.1 \pm 13.9$ & 0.11 \\
Relative wall thickness & $0.41 \pm 0.05$ & $0.43 \pm 0.05$ & 0.09 \\
Ejection fraction, \% & $61.5 \pm 2.3$ & $62.3 \pm 2.5$ & 0.42 \\
LA volume index, mL/m ${ }^{2}$ & $23.9 \pm 4.1$ & $34.5 \pm 4.6$ & 0.01 \\
Mitral E velocity, cm/s & $75 \pm 13$ & $71 \pm 11$ & 0.09 \\
Mitral A velocity, cm/s & $73 \pm 11$ & $72 \pm 13$ & 0.13 \\
E/A ratio & $1.1 \pm 0.3$ & $0.9 \pm 0.1$ & 0.07 \\
Mean E/é & $7.5 \pm 1.3$ & $11.3 \pm 2.0$ & $<0.001$ \\
PA lateral-PA tricuspid, ms & $31.5 \pm 9.8$ & $43.5 \pm 12.0$ & $<0.001$ \\
PA lateral-PA septum, ms & $23.6 \pm 7.5$ & $29.4 \pm 11.8$ & $<0.001$ \\
PA septum-PA tricuspid, ms & $11.3 \pm 5.2$ & $14.5 \pm 4.4$ & $<0.01$ \\
\hline
\end{tabular}

Data are presented as mean \pm SD. LVMI, left ventricular mass index; LA, left atrial; PA, time interval from $\mathrm{P}$ wave in ECG to A wave in mitral flow velocity.

Table 4. Association between blood variability index $\left(\mathrm{SD}_{\mathrm{dn}}\right)$ and echocardiographic variables in multiple linear analysis

\begin{tabular}{lcc}
\hline & Correlation coefficient & $p$ value \\
\hline Ejection fraction, \% & -0.028 & 0.53 \\
LAVi, mL/m & 0.139 & $<0.05$ \\
E/e' ratio & 0.175 & $<0.03$ \\
Intra-LA EMD & 0.392 & $<0.001$ \\
Inter-atrial EMD & 0.261 & $<0.01$ \\
Intra-right atrial EMD & 0.231 & $<0.01$ \\
\hline
\end{tabular}

LAVi, left atrial volume index; LA, left atrial; EMD, electromechanical delay.

Table 5. Cox regression analysis of weighted systolic blood pressure variability index $\left(\mathrm{SD}_{\mathrm{dn}}\right)$ for predicting NOAF

\begin{tabular}{lccc}
\hline Variable & HR & $95 \% \mathrm{CI}$ & $p$ values \\
\hline Age, years & 0.97 & $0.91-1.02$ & 0.79 \\
Gender & 0.95 & $0.17-1.81$ & 0.38 \\
Hypertension & 0.98 & $0.65-1.33$ & 0.09 \\
Diabetes mellitus & 1.13 & $0.09-2.11$ & 0.21 \\
Dyslipidemia & 0.65 & $0.15-1.07$ & 0.63 \\
Smoking & 1.25 & $0.39-2.16$ & 0.60 \\
Ejection fraction, $\%$ & 0.68 & $0.21-1.25)$ & 0.45 \\
LAVi, mL/m ${ }^{\prime}$ & 1.15 & $(0.43-1.98)$ & 0.17 \\
E/e' ratio & 1.30 & $(0.23-2.41)$ & 0.11 \\
BPV index $\left(\mathrm{SD}_{\mathrm{dn}}\right) \geq 12.8 \mathrm{~mm} \mathrm{Hg}$ & 3.75 & $(1.59-6.35)$ & $<0.001$ \\
Atrial EMD & 2.72 & $0.95-5.03$ & $<0.001$ \\
\hline
\end{tabular}

LAVi, left atrial volume index; BPV, blood pressure variability; EMD, electromechanical delay. 
Mahfouz et al.: Blood Pressure Variability and Atrial Fibrillation in STEMI

Fig. 1. ROC curve of the optimal cut-off value to predict new-onset atrial fibrillation in patients with STEMI.

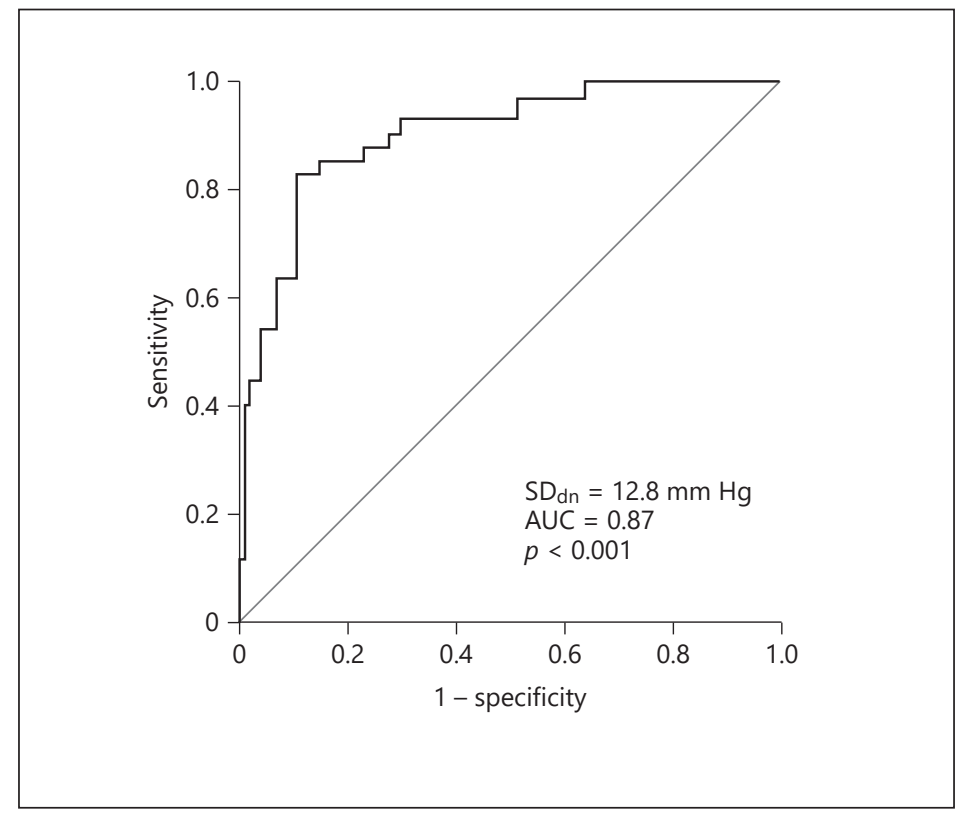

increased in the group with high BPV, whereas LV mass index, relative wall thickness, and $\mathrm{EF} \%$ were comparable. Importantly, the parameters of atrial EMD were significantly increased in patients with high BVP compared with those with low BPV ( $p<0.001$ for all).

With correlation analysis (Table 4), there was significant positive correlation between $\mathrm{SD}_{\mathrm{dn}} \operatorname{LAVi}(p<0.05), \mathrm{E} / \mathrm{e}^{\prime}(p<0.03)$, intra-LA EMD $(p<0.001)$ and inter-atrial EMD $(p<0.01)$. Cox regression analysis (Table 5) revealed that $\mathrm{SD}_{\mathrm{dn}}$ of systolic $\mathrm{BP}$ and intra-atrial EMD were independent predictors for NOAF during follow-up in patients with STEMI (OR $=3.75$ and 02.72; $p<0.001$ for both).

Using ROC curves (Fig. 1), the BPV index (weighted $\mathrm{SD}_{\mathrm{dn}}$ ) value of $12.8 \mathrm{~mm} \mathrm{Hg}$ was the optimal cut-off value to predict the development of NOAF in patients with STEMI; AUC $=0.87$ ( $95 \%$ confidence limit, $0.79-0.95$ ), with a sensitivity of $81 \%$ and specificity of $93 \%$.

\section{Discussion}

Our study was designed to investigate the association between in-hospital short-term BPV in patients with STEMI and NOAF during a 1-year follow-up in relation to atrial EMD. The results confirmed that intra-left atrial and inter-atrial EMD were increased in STEMI patients with in-hospital short-term BPV. Furthermore, we found that systolic, not diastolic, BPV determines the development of NOAF during follow-up, in both hypertensive and nonhypertensive patients. Importantly, we found that systolic BPV index value $\geq 12.8 \mathrm{~mm} \mathrm{Hg}$ is the best cut-off value to predict the incidence of NOAF during long-term follow-up.

Previous studies have clearly shown that enhanced fluctuation of BP induces more vascular stiffness and artery remodeling, and may be the spark for the initiation of arteriosclerotic disease [18]. Many clinical trials have demonstrated that short- and long-term BPV not only independently contribute to cerebrovascular events but also implicate prognostic significance [7, 19-21].

The pathogenesis of the independent arrhythmogenic effect of BPV in the setting of STEMI is not clear and it is not obvious whether BPV would help to predict NOAF. 
In the setting of acute STEMI, patients may have autonomic instability, which could be associated with BP fluctuation. In addition, those patients may have left atrial remodeling due to diastolic dysfunction and increased left atrial wall tension. These factors may trigger the development of atrial fibrillation.

The mechanism by which BPV may be associated with atrial EMD in the setting of STEMI is not clearly understood. However, in STEMI patients, increased LAVi, diastolic dysfunction, and left atrial remodeling may be associated with elevated brain natriuretic peptide due to increased wall tension - a finding that may be associated with both atrial fibrillation [22] and BPV [23].

It is clearly thought that the autonomic nervous system has a significant role in both short-term BP regulation and long-term sympathetic overactivity. This is suggested to be a mechanistic link between coronary artery disease and atrial fibrillation [24].

It is suggested that autonomic instability, chronic left atrial remodeling, and diastolic LV dysfunction may have a significant impact on the occurrence of NOAF. Furthermore, STEMI patients usually have neurohumoral activation [25] and cardiovascular inflammation [26] pathophysiologic changes that may play a significant role in both BPV and increased atrial EMD.

With multivariate analysis, the results indicated that BPV and atrial EMD were independent predictors of the occurrence of NOAF during follow-up.

Konstantinou et al. [27] demonstrated that in patients with acute coronary syndrome, in-hospital systolic BPV was associated with adverse cardiovascular events during the 6 months of follow-up. This finding could be related to autonomic dysfunctional status associated with the pathophysiology of acute coronary syndrome, linking BP regulation mechanisms to adverse events.

In our study, increased atrial EMD, a marker of delayed atrial conduction in STEMI patients, was associated with BPV. The delayed atrial conduction suggests that electrophysiological changes in STEMI patients is more evident in subjects with BPV.

\section{Limitations}

First, the sample size was small. Second, this was a single-center study. Third, and importantly, the ABPM study was limited to $24 \mathrm{~h}$.

\section{Conclusion}

Our findings showed that short-term BPV is associated with NOAF in patients with STEMI. Short-term BPV is predicted to be a contributor factor for left atrial EMD. These findings may explain the development of atrial fibrillation during follow-up of patients with STEMI.

\section{Statement of Ethics}

All participants gave a written informed consent, and the study was approved by the faculty ethical and scientific committee according to the Helsinki Declaration.

\section{Disclosure Statement}

The authors declare that they have no conflict of interest.

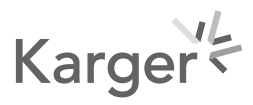




\begin{tabular}{l|l}
\hline Pulse 2020;8:57-65 \\
\hline DOI: 10.1159/000507792 & $\begin{array}{l}\text { @ 2020 S. Karger AG, Basel } \\
\text { www.karger.com/pls }\end{array}$ \\
\hline
\end{tabular}

Mahfouz et al.: Blood Pressure Variability and Atrial Fibrillation in STEMI

\section{Funding Sources}

This study did not receive any specific funding.

\section{Author Contributions}

Ragab A. Mahfouz: idea, design of the study, and final approval. Mohamed El-Shetry and Mohamed Safwat: conception, collection of the data, and preparing the manuscript. Abdelfattah Frere: analysis.

\section{References}

1 Batra G, Svennblad B, Held C, Jernberg T, Johanson P, Wallentin L, et al. All types of atrial fibrillation in the setting of myocardial infarction are associated with impaired outcome. Heart. 2016 Jun;102(12):926-33.

2 Jons C, Jacobsen UG, Joergensen RM, Olsen NT, Dixen U, Johannessen A, et al.; Cardiac Arrhythmias and Risk Stratification after Acute Myocardial Infarction (CARISMA) Study Group. The incidence and prognostic significance of new-onset atrial fibrillation in patients with acute myocardial infarction and left ventricular systolic dysfunction: a CARISMA substudy. Heart Rhythm. 2011 Mar;8(3):342-8.

3 Aronson D, Mutlak D, Bahouth F, Bishara R, Hammerman H, Lessick J, et al. Restrictive left ventricular filling pattern and risk of new-onset atrial fibrillation after acute myocardial infarction. Am J Cardiol. 2011 Jun; 107(12):1738-43.

4 Herpin D, Ragot S. [Variability of blood pressure. Clinical and therapeutic implications]. Rev Med Interne. 1995;16(2):131-6. French.

5 Rothwell PM, Howard SC, Dolan E, O’Brien E, Dobson JE, Dahlöf B, et al. Prognostic significance of visit-to-visit variability, maximum systolic blood pressure, and episodic hypertension. Lancet. 2010 Mar;375(9718):895905.

6 Verdecchia P, Reboldi G, Angeli F, Trimarco B, Mancia G, Pogue J, et al. Systolic and diastolic blood pressure changes in relation with myocardial infarction and stroke in patients with coronary artery disease. Hypertension. 2015 Jan;65(1):108-14.

7 Muntner P, Whittle J, Lynch AI, Colantonio LD, Simpson LM, Einhorn PT, et al. Visit-to-visit variability of blood pressure and coronary heart disease, stroke, heart failure, and mortality: a cohort study. Ann Intern Med. 2015 Sep;163(5):329-38.

8 Omi W, Nagai H, Takamura M, Okura S, Okajima M, Furusho H, et al. Doppler tissue analysis of atrial electromechanical coupling in paroxysmal atrial fibrillation. J Am Soc Echocardiogr. 2005 Jan;18(1):39-44.

9 Ozer N, Yavuz B, Can I, Atalar E, Aksöyek S, Ovünç K, et al. Doppler tissue evaluation of intra-atrial and interatrial electromechanical delay and comparison with P-wave dispersion in patients with mitral stenosis. J Am Soc Echocardiogr. 2005 Sep;18(9):945-8.

10 Thygesen K, Alpert JS, Jaffe AS, Simoons ML, Chaitman BR, White HD; Writing Group on behalf of the Joint ESC/ ACCF/AHA/WHF Task Force for the Universal Definition of Myocardial Infarction. Third universal definition of myocardial infarction. Glob Heart. 2012 Dec;7(4):275-95.

11 Jain S, Ting HT, Bell M, Bjerke CM, Lennon RJ, Gersh BJ, et al. Utility of left bundle branch block as a diagnostic criterion for acute myocardial infarction. Am J Cardiol. 2011 Apr;107(8):1111-6.

12 Head GA, McGrath BP, Mihailidou AS, Nelson MR, Schlaich MP, Stowasser M, et al. Ambulatory blood pressure monitoring in Australia: 2011 consensus position statement. J Hypertens. 2012 Feb;30(2):253-66.

13 Bilo G, Giglio A, Styczkiewicz K, Caldara G, Maronati A, Kawecka-Jaszcz K, et al. A new method for assessing 24-h blood pressure variability after excluding the contribution of nocturnal blood pressure fall. J Hypertens. 2007 Oct;25(10):2058-66.

14 Staessen JA, Bieniaszewski L, O'Brien E, Gosse P, Hayashi H, Imai Y, et al. Nocturnal blood pressure fall on ambulatory monitoring in a large international database. The "Ad Hoc' Working Group. Hypertension. 1997 Jan;29(1 Pt 1):30-9.

15 Marfella R, Gualdiero P, Siniscalchi M, Carusone C, Verza M, Marzano S, et al. Morning blood pressure peak, QT intervals, and sympathetic activity in hypertensive patients. Hypertension. 2003 Feb;41(2):237-43.

16 Lang RM, Badano LP, Mor-Avi V. Recommendations for cardiac chamber quantification by echocardiography in adults: an update from the American Society of Echocardiography and the European Association of Cardiovascular Imaging. J Am Soc Echocardiogr. 2015;28:1-39.e14.

17 Deniz A, Sahiner L, Aytemir K, Kaya B, Kabakci G, Tokgozoglu L, et al. Tissue Doppler echocardiography can be a useful technique to evaluate atrial conduction time. Cardiol J. 2012;19(5):487-93.

18 Nagai M, Dote K, Kato M, Sasaki S, Oda N, Kagawa E, et al. Visit-to-visit blood pressure variability and classes of antihypertensive agents; associations with artery remodeling and the risk of stroke. Curr Pharm Des. 2016; 22(3):383-9.

19 Manning LS, Rothwell PM, Potter JF, Robinson TG. Prognostic significance of short-term blood pressure variability in acute stroke: systematic review. Stroke. 2015 Sep;46(9):2482-90.

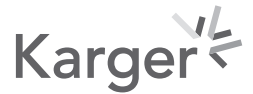


20 Manning LS, Mistri AK, Potter J, Rothwell PM, Robinson TG. Short-term blood pressure variability in acute stroke: post hoc analysis of the controlling hypertension and hypotension immediately post stroke and continue or stop post-stroke antihypertensives collaborative study trials. Stroke. 2015 Jun; 46(6):1518-24.

21 Yamada T, Fukunami M, Shimonagata T, Kumagai K, Ogita H, Asano Y, et al. Prediction of paroxysmal atrial fibrillation in patients with congestive heart failure: a prospective study. J Am Coll Cardiol. 2000 Feb;35(2): 405-13.

22 Rodrigues AB, Gismondi RA, Lagoeiro A, Cecilio AM, Vasques D, Arita R, et al. Association between B-type natriuretic peptide and within-visit blood pressure variability. Clin Cardiol. 2018 Jun;41(6):778-81.

23 Bruno RM, Ghiadoni L, Seravalle G, Dell'oro R, Taddei S, Grassi G. Sympathetic regulation of vascular function in health and disease. Front Physiol. 2012 Jul;3:284.

24 Lieder H, Breithardt G, Heusch G. Fatal attraction - A brief pathophysiology of the interaction between atrial fibrillation and myocardial ischemia. Int J Cardiol. 2018 Mar;254:132-5.

25 Kato J, Kitamura K, Uemura T, Kuwasako K, Kita T, Kangawa K, et al. Plasma levels of adrenomedullin and atrial and brain natriuretic peptides in the general population: their relations to age and pulse pressure. Hypertens Res. 2002 Nov; 25(6):887-92.

26 Chae CU, Lee RT, Rifai N, Ridker PM. Blood pressure and inflammation in apparently healthy men. Hypertension. 2001 Sep;38(3):399-403.

27 Konstantinou K, Tsioufis K, Dimitriadis K, et al. Reduced blood pressure variability as a predictor of cardiac events after myocardial infarction. J Hypertens. $2018 \mathrm{Jun}$;6:e12. Available from: https://doi.org/10.1097/01. hjh.0000538993.55964.b8. 